\title{
Biochemical and haematological profile of donkeys in the Czech and Slovak Republics: influence of age and sex
}

\author{
Markéta Sedlinská ${ }^{1}$, Eliška Horáčková $^{1}$, Michal Vyvial ${ }^{1}$, Miroslava Mráčková ${ }^{1}$, Eva Jánová ${ }^{2}$ \\ University of Veterinary and Pharmaceutical Sciences Brno, Faculty of Veterinary Medicine, \\ ${ }^{1}$ Equine Clinic, ${ }^{2}$ Department of Animal Genetics, Central European Institute of Technology, \\ Brno, Czech Republic \\ Received December 5, 2016 \\ Accepted May 31, 2017
}

\begin{abstract}
The aim of this study was to evaluate the importance of taking into account the age and sex of the donkeys while assessing individual haematological and biochemical variables. Material used for the study were blood samples collected from 112 clinically healthy donkeys ( 37 males and 75 females) aged from 05 to 30 years old. Selected haematological and biochemical variables were examined and the influence of sex and age was analyzed by multivariate analysis. Significant differences were found in some of biochemical and haematological variables between groups. The results of this study can be useful for appropriate clinical interpretation of laboratory results in donkeys.
\end{abstract}

Blood analysis, serum analysis, jenny, jack, foals

Biochemical and haematological examination of the blood is routinely used in preventive medicine, diagnosis of disease and verification of treatment success in all kinds of animals. Individual species have specific physiological ranges of blood variables, donkeys included. Zinkl et al. (1990) and Jordana et al. (1998) proved that donkey laboratory findings are not completely comparable with reference to other species nor even to, their closest relatives, horses.

Scientific reports show that a large number of factors can affect blood biochemical and haematological indices even within one species. Changes due to geographic location, nutritional status or the effect of management practice have already been proved (Jordana et al. 1998; Mushi et al. 1999; Sow et al. 2012; Sedlinska et al. 2016).

The influence of the donkey's age and sex has also already been reported (Folch et al. 1997; Jordana et al. 1998; Mori et al. 2003, 2004; Girardi et al. 2013, 2014). On the other hand, there is a study which investigated about 4000 donkeys without proof that biochemical variables are affected by the age and sex (French and Patrick 1995).

A previous study by Sedlinska et al. (2016) established normal reference values of biochemical and hematological indices of donkeys in the Czech and Slovak Republics (Central Europe); those values can be useful for clinical routines, allowing veterinarians to establish appropriate interpretations of laboratory data. This study aims to evaluate how important it is to take into account the donkey's age and sex while assessing individual haematological and biochemical variables.

\section{Materials and Methods}

Blood serum collected from 112 clinically healthy donkeys (37 males and 75 females) aged from 0.5 to 30 years and housed in different locations and conditions ( 26 different owners) in the Czech and Slovak Republics were examined for selected haematological and biochemical indices.

Blood was collected by jugular venipuncture into $10 \mathrm{ml}$ tubes for biochemistry and $2.5 \mathrm{ml}$ ethylenediamine tetraacetic acid (EDTA) tubes for haematology. The haematological indices examined were: red blood cells

Address for correspondence:

MVDr. Markéta Sedlinská, Ph.D.

Equine Clinic, Faculty of Veterinary Medicine

University of Veterinary and Pharmaceutical Sciences Brno

Palackého tř. 1946/1, 612 42, Brno, Czech Republic 


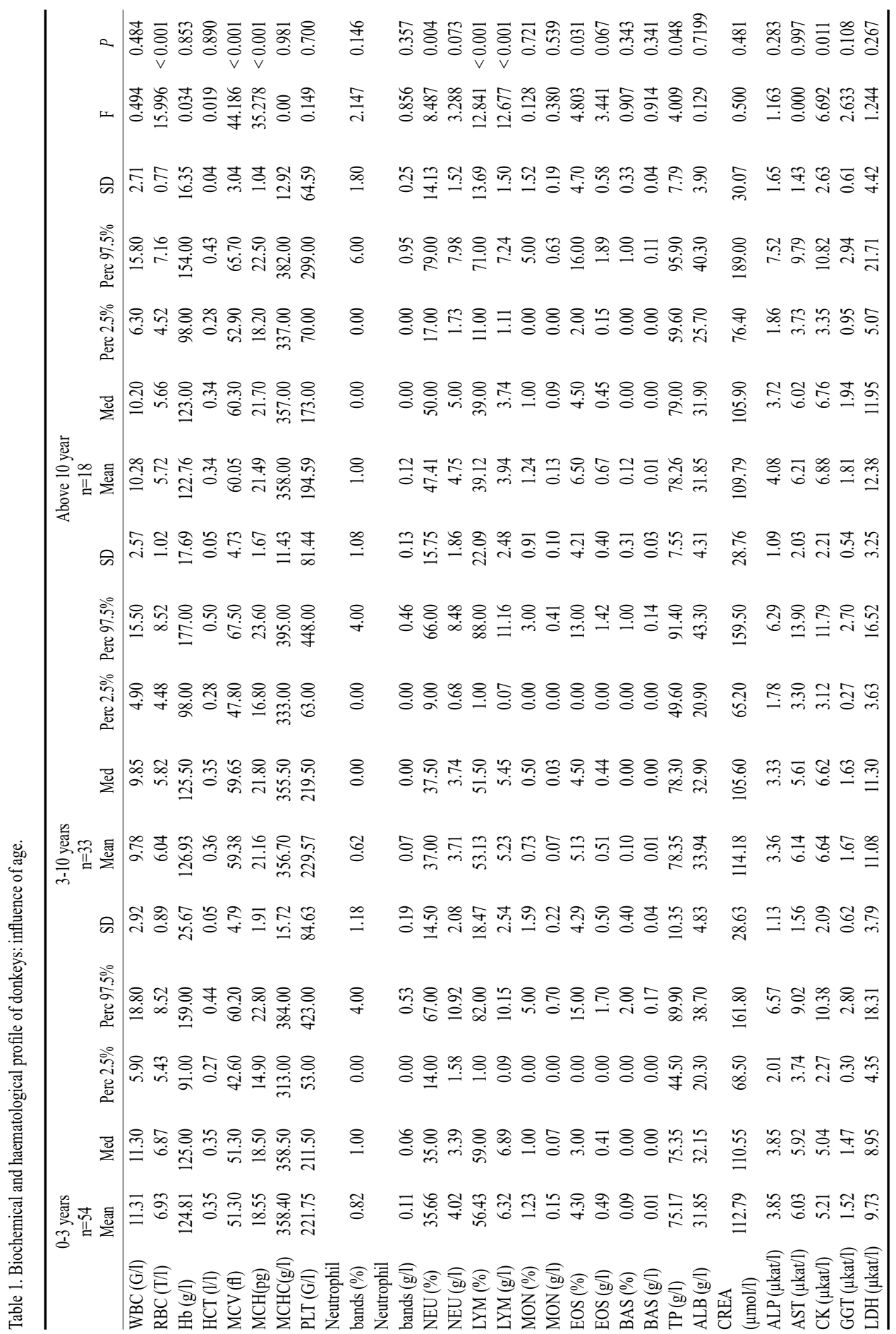




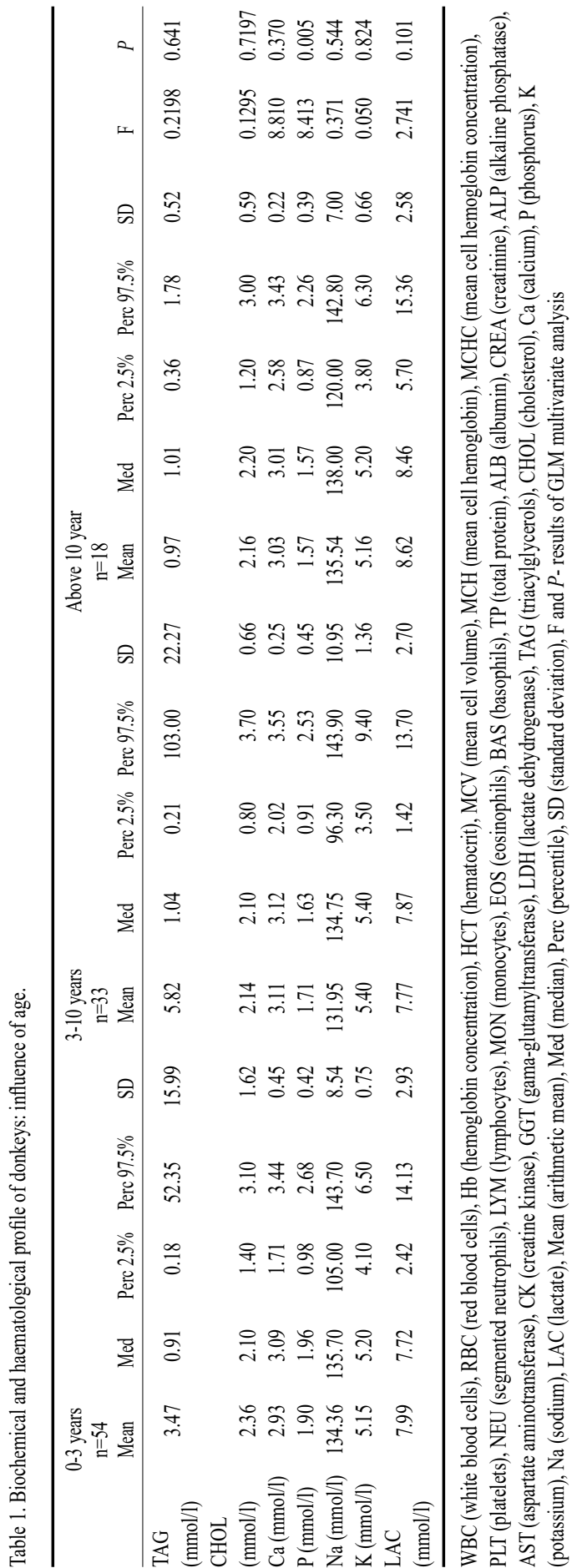

(RBC), white blood cells (WBC), haemoglobin concentration $(\mathrm{Hb})$, haematocrit (HCT), mean cell volume (MCV), mean cell haemoglobin $(\mathrm{MCH})$, mean cell haemoglobin concentration (MCHC), platelets (PLT), segmented neutrophils (NEU), neutrophil bands, lymphocytes (LYM), monocytes (MON), eosinophils (EOS), and basophils (BAS). Indices were calculated using the heamatological analyzer Celltac alpha MEK 6318 (Nihon Kohden, Tokyo, Japan). For each sample, a peripheral blood smear from fresh blood was prepared, stained by the Diff-Quick method and a differential leukocyte count was examined microscopically.

Samples for biochemistry were allowed to clot at room temperature and serum was separated by centrifugation at $3000 \mathrm{~g}$ for $15 \mathrm{~min}$. Serum samples were analyzed for metabolites: total protein (TP), albumin (ALB), creatinine (CREA), alkaline phosphatase (ALP), aspartate aminotransferase (AST), creatine kinase (CK), gama-glutamyltransferase (GGT), lactate dehydrogenase (LDH), triacylglycerols (TAG), cholesterol (CHOL), calcium (Ca), phosphorus $(\mathrm{P})$, potassium $(\mathrm{K})$, sodium $(\mathrm{Na})$ and lactate (LAC). All analyses were performed at the Central Clinical Laboratory of the University of Veterinary and Pharmaceutical Sciences Brno by the biochemical analyzer DPC Konelab $20 \mathrm{i}^{\circledR}$ (Thermo Fisher Scientific Oy, Vantaa, Finland).

The effects of age (continuous variable) and sex (categorical variable) on blood indices were analyzed by multivariate analysis (GLM) in the software Statistica for Windows.

\section{Results}

The results of biochemical and haematological profiling are listed in Table 1. The results of biochemical and haematological profiling divided according to the sex (male or female donkey) are listed in Table 2. In the study, some significant differences $(P<0.05)$ were found: RBC, MCV, $\mathrm{MCH}, \mathrm{NEU}, \mathrm{LYM}$, EOS, CK, serum phosphorus in relation to age, and $\mathrm{MCV}$, $\mathrm{MCH}, \mathrm{TP}, \mathrm{CREA}$ in relation to sex.

\section{Discussion}

In relation to age, significant differences were found in the following variables: $\mathrm{RBC}, \mathrm{MCV}, \mathrm{MCH}, \mathrm{NEU}$, LYM, EOS, CK, and serum phosphorus.

The significant decrease of RBC with age, which was found in this study, had also been described previously 


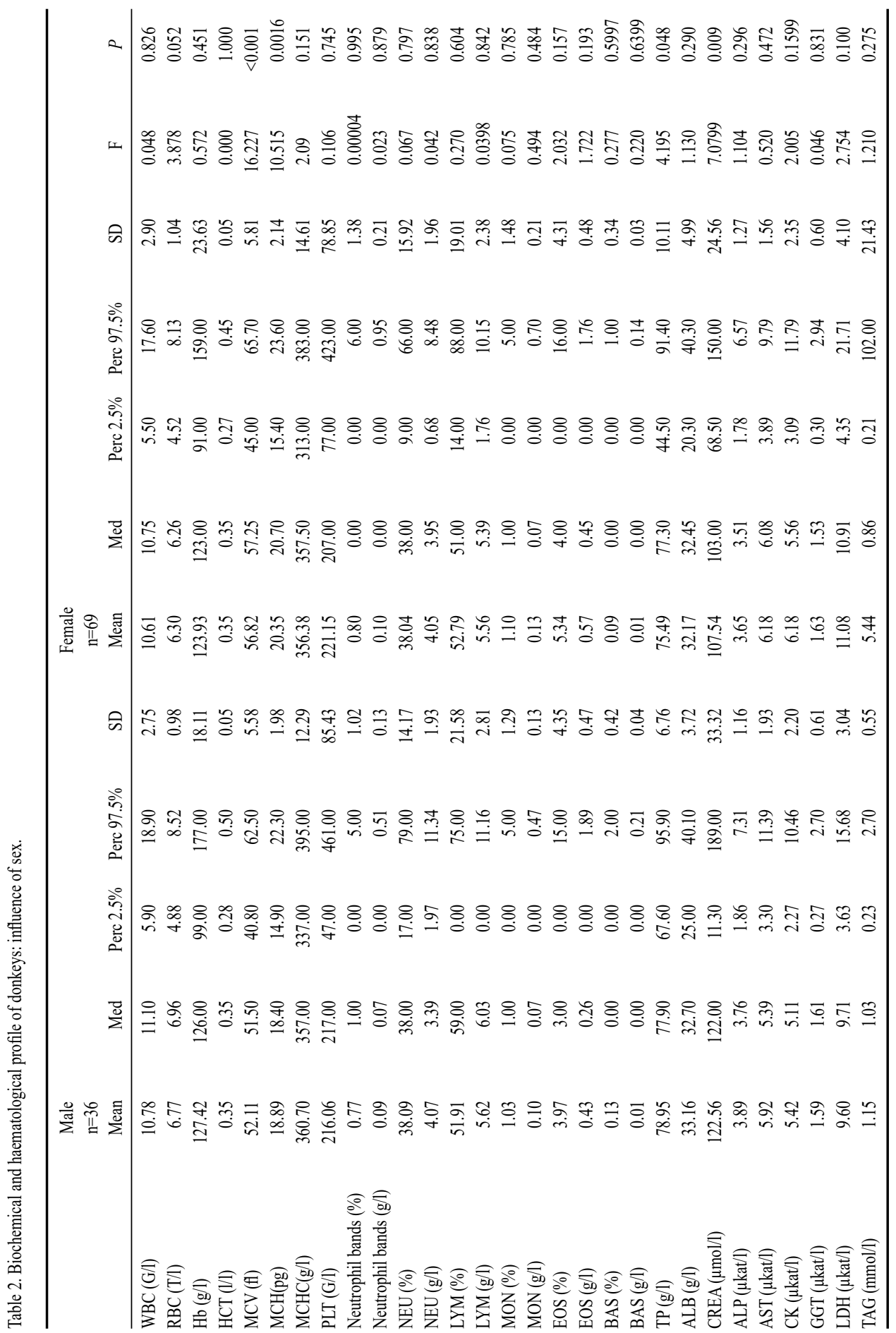




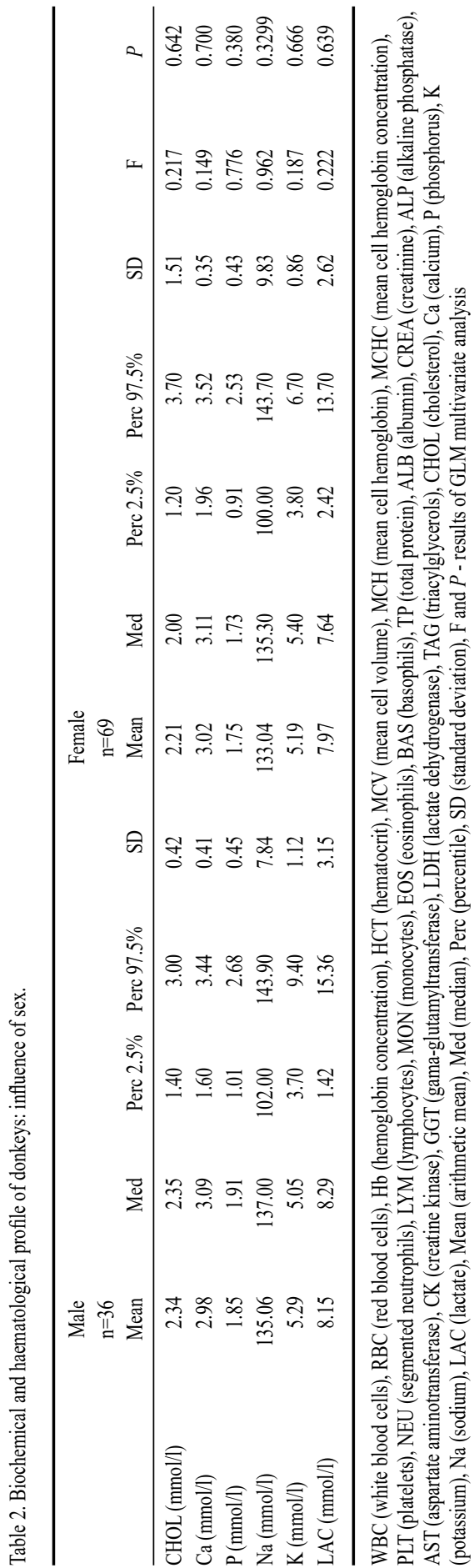

by several other authors (Zinkl et al. 1990; Folch et al. 1997; Caldin et al. 2005; Laus et al. 2015). Some previous studies (Zink1 et al. 1990; Caldin et al. 2005; Laus et al. 2015) reported this alteration as associated with the smaller size of red cells in young donkeys that they explained by a slight deficiency of iron. In this study, significantly lower values of $\mathrm{MCV}$ and $\mathrm{MCH}$ were found in donkeys under 3 years old, but the iron level was not measured.

A significantly lower content of LYM was also found in donkeys older than 10 years, which is similar to that reported by Folch et al. (1997).

The NEU values in this study were higher in donkeys older than 10 years, which is not in agreement with previous reports by Folch et al. (1997) and Caldin et al. (2005) who reported a reduction of this variable with aging.

The EOS in our study tended to increase with age, which is in agreement with other studies (Zinkl et al. 1990; Girardi et al. 2014) but at odds with Folch et al. (1997) who reported a decrease of this variable with aging. We can presume that this increase in EOS may be attributed to a higher parasitic burden with age or progressive contact with allergen antigens.

The influence of age on the biochemical properties was revealed. The level of TP was greater for older animals, which is similar to other studies, where an increase level of total protein with age was described (Zinkl et al. 1990; Folch et al. 1997; Girardi et al. 2013), possibly due to the increasing amount of globulins.

This study found an increasing content of CK associated with aging. This can be due to the difference in muscle mass (due to different exercise) between the studied groups. However, no effect of age on CK was found in other studies (French and Patrick 1995; Girardi et al. 2013).

This study also found a significant difference in the values of plasmatic phosphorus concentrations in relation to age. Significantly higher values were found in donkeys up to 3 years of age, which confirmed previous studies (Zinkl et al. 1990; Jordana et al. 1998; Caldin et al. 2005; Girardi et al. 2013). This is probably due to an increased bone metabolism in younger animals. 
In relation to sex, significant differences were found in the following indicators: $\mathrm{MCV}$, $\mathrm{MCH}, \mathrm{TP}$, and CREA. In this study significantly higher $(P<0.05)$ values of MCH and MCV were found in female animals. This is in complete contrast to the results of the study by Babeker and Bdalbagi (2014) who found significantly higher values of $\mathrm{MCH}$ and $\mathrm{MCHC}$ in male donkeys. Their study also reports significantly lower values of $\mathrm{Hb}$ in female donkeys and slightly higher levels of RBC in male animals, which was also confirmed in our study. The lower RBC in females was probably because female oestrogens decrease erythropoietin production.

Girardi et al. (2013) report that males show greater values of CREA. This is also in agreement with our study where males have higher CREA. This can be due to bigger muscle tissue in males compared to females.

Data in our study showed significant differences in several biochemical and haematological analytes between groups of 112 healthy donkeys divided by age and sex. This analysis can help with future investigations on the influence of age and sex on haematological and biochemical indices under different conditions. An appropriate interpretation of laboratory results can help to improve the veterinary care of donkeys. However, there are some discrepancies in this field. On the one hand, many previous studies (mentioned above) describe varying indices for different sexes and ages in a population of donkeys (Folch at al. 1997; Jordana et al. 1998; Mori et al. 2003, 2004; Girardi et al. 2013, 2014). On other hand, in the study of French and Patrick (1995) based on a population of approximately 4,000 donkeys, no differences in biochemical indices in relation to age and sex were found. Additionally, Laus et al. (2015) found no differences between sexes in biochemical and haematological properties, and Yakubu and Chafe (2008) found no correlation between the haematological indices and sex. More investigation in this field is needed.

\section{References}

Babeker EA, Bdalbagi YMA 2014: Hematological profile of donkey (Equus asinus) breed in White Nile State, Sudan. University of Bakht Alruda Scientific Journal 10: 218-228

Caldin M, Furlanello T, Solano-Gallego L, De Lorenzi D, Carli E, Tasca S, Lubas G 2005: Reference ranges for haematology, biochemical profile and electrophoresis in a single herd of Ragusana donkeys from Sicily (Italy). Comp Clin Pathol 14: 5-12

Folch P, Jordana J, Cuenca R 1997: Reference ranges and the influence of age and sex on haematological values of the endangered Catalonian donkey. Vet J 154: 163-168

French, JM and Patrick, VH 1995: Reference values for physiological, haematological and biochemical parameters in domestic donkeys (Equus asinus). Equine Vet Educ 7: 33-35

Girardi AM, Marques LC, De Toledo CZP, Barboso JC, Maldonado Jr, W, Jorge RLN, Da Silva Nogueira CA 2013: Biochemical profile of the Pêga donkey (Equus asinus) breed: influence of age and sex. Comp Clin Pathol 23: 941-947

Girardi AM, Marques LC, De Toledo CZP, De Campos EF 2014: Hematological variables of the Pêga donkey (Equus asinus) breed: influence of age and sex. Comp Clin Pathol 24: 335-342

Jordana J, Folch P, Cuenca R 1998: Clinical biochemical parameters of the endangered Catalonian donkey breed: normal values and the influence of sex, age, and management practices effect. Res Vet Sci 64: 7-10

Laus F, Spaterna A, Faillace V, Paggi E, Serri E, Vullo C, Cerquetella M, Tesei B 2015: Reference for hematological and biochemical parameters of mixed breed donkeys (Equus asinus). Wulfenia J 22: 294-304

Mori E, Fernandes WR, Mirandola RMS, Kubo G, Ferreira RR, Oliveira JV, Gacek F 2003: Reference values on serum biochemical parameters of Brazilian donkey (Equus asinus) breed. J Equine Vet Sci 23: 358-364

Mori E, Mirandola RMS, Ferreira RR, Oliveira JV, Gacek F, Fernandes WR 2004: Reference values on hematologic parameters of the Brazilian donkey (Equus asinus) breed. J Equine Vet Sci 24: 271-276

Mushi EZ, Binta MG, Ndebele RT 1999: Hematological studies on apparently healthy donkeys in Oodi, Kgatleng district Botswana. Proceedings of the workshop of the Animal Traction Network for Eastern and Southern Africa (ATNESA) held 20-24, Mpumalanga, South Africa: 344 p., http://www.atnesa.org/

Sedlinská M, Biricová D, Horáčková E, Mráčková M 2016: Selected haematological and biochemical indices in donkeys in the Czech and Slovak Republics. Acta Vet Brno 85: 227-230

Sow A, Kalandi KM, Ndiaye NP, Bathily A, Sawadogo GJ 2012: Clinical biochemical parameters of Burkinabese local donkeys' breeds. IRJBB 2: 84-89 
Yakubu, AS and Chafe, UM 2008: Haematological studies of donkeys in Sokoto state, Nigeria. Sokoto J Vet Sci 7: 41-45

Zinkl GJ, Mae D, Merida PG, Forva TB, Humble JA 1990: Reference ranges and the influence of age and sex on hematologic and serum biochemical values in donkeys (Equus asinus). Am J Vet Res 51: 408-413 